\title{
Luminous Blue Variables, cool hypergiants and some impostors in the H-R diagram
}

\author{
Roberta M. Humphreys \\ Department of Astronomy, University of Minnesota, \\ 116 Church Street SE, Minneapolis, MN55455, USA
}

\begin{abstract}
Current observations of the SDor/LBVs and candidates and the implications for their important role in massive star evolution are reviewed. Recent observations of the cool hypergiants are altering our ideas about their evolutionary state, their atmospheres and winds, and the possible mechanisms for their asymmetric high mass loss episodes which may involve surface activity and magnetic fields. Recent results for IRC+10420, $\rho$ Cas and VYCMa are highlighted. S Dor/LBVs in eruption, and the cool hypergiants in their high mass loss phases with their optically thick winds are not what their apparent spectra and temperatures imply; they are then 'impostors' on the H-R diagram. The importance of the very most massive stars, like $\eta$ Carinae and the 'supernovae impostors' are also discussed.
\end{abstract}

\section{Introduction}

The Luminous Blue Variables and the cool hypergiants represent two high mass loss, very unstable stages near the ends of massive star evolution. These two groups of evolved massive stars are our best candidates for investigating and understanding the physical cause of the empirical upper luminosity boundary in the H-R diagram (Humphreys \& Davidson 1979, 1984).

These two very different groups of stars are connected, or disconnected, via their evolution. The classical LBVs or SDoradus variables (Humphreys \& Davidson 1994; Humphreys 1999) have presumably evolved from stars with masses $z 50-60 \mathrm{M}_{\odot}$ and are at or past the end of core H-burning. They have not, and apparently will not, become red supergiants, but instead, via high mass loss episodes, it is thought that they will evolve directly to the Wolf-Rayet stage. The less luminous LBVs have initial masses $\lesssim 40 \mathrm{M}_{\odot}$ and have probably already been through the red supergiant stage. With their lowered $g_{\text {eff }}$ as post-red supergiants, they will be more subject to atmospheric instabilities. I describe the cool hypergiants as the very luminous and often highly unstable post-mainsequence stars near the upper luminosity boundary with apparent temperatures $\lesssim 10000 \mathrm{~K}$ and spectral types A to M. These stars are currently RSGs or are in the process of entering or leaving the red supergiant region. Indeed one of the issues I will address in this review is the direction of evolution of the 'yellow' hypergiants.

But in addition to the questions of evolution, there is also an astrophysical connection at the opaque wind limit -7000 to $8000 \mathrm{~K}$ - where the LBVs 


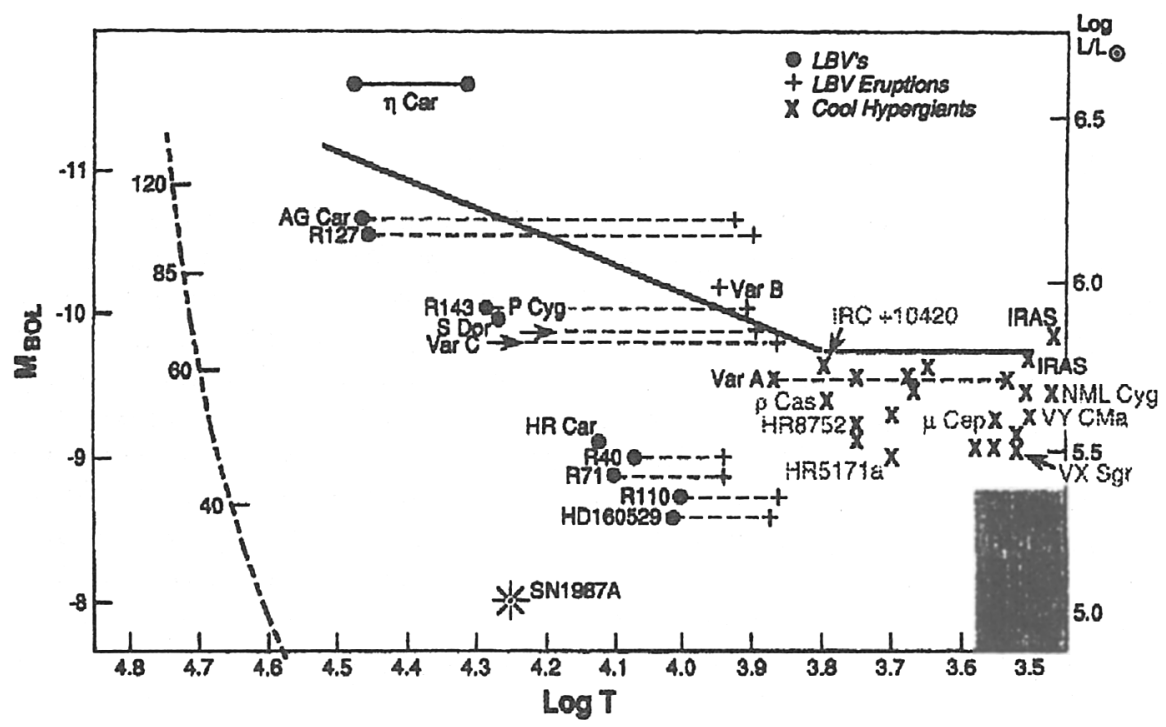

Figure 1. A schematic H-R diagram showing the location of the SDoradus/Luminous Blue Variables and the cool hypergiants. The strong solid line is the empirical upper luminosity boundary.

in eruption or outburst produce a slow, dense, cool wind at their visual maximum light (Figure 1). I will show that hypergiants evolving blueward from the RSG stage may also encounter this opaque wind limit, as demonstrated by the post-RSG IRC+10 420 (Jones et al. 1993; Humphreys et al. 1997; Oudmaijer et al. 1996; Humphreys et al. 2002).

\section{S Doradus or Luminous Blue Variables}

The LBV terminology was first introduced by Conti (1984) and it initially served us well by acknowledging the astrophysical similarities among groups of stars that were known variously in the literature as S Dor variables, Hubble-Sandage variables, $\mathrm{P}$-Cygni stars and $\eta$ Carinae variables. However, it is increasingly apparent that many evolved massive, hot (blue) stars are somewhat variable. Therefore at the 1998 meeting (Variable and Non-spherical Stellar Winds in Luminous Hot Stars, 1999, eds. B. Wolf, O. Stahl \& A.W. Fullerton), I (Humphreys 1999) suggested that we return to the SDoradus designation. This designation describes a certain type of variability in which a hot star typically brightens 1 - 2 magnitudes in the visible, and at constant luminosity, its apparent temperature appears to cool as its envelope approaches the opaque wind limit (Davidson 1987) in an 'eruption' or outburst.

There has been an abundance observations and analysis of SDor type variables and candidate LBVs in recent years that it is not possible to mention all of them in this brief review. Instead I will draw attention to some recent reviews and conference volumes with numerous papers about these stars:

- a recent review of S Doradus variability by van Genderen, 2001;

- P Cygni 2000, 400 Years of Progress, 2001, edited by de Groot \& Sterken; 
- Eta Carinae and Other Mysterious Stars ..., 2001, edited by Johansson, Gull \& Davidson.

\subsection{The census of LBVs/S Dor Variables}

In our 1994 review article (Humphreys \& Davidson 1994), we listed 30 confirmed S Dor/LBVs plus eight candidates in various galaxies, mostly in the Local Group. I want to add Romano's star in M 33 to the list of confirmed members. Kurtev et al. (2001) have shown that it has varied $\sim 1$ mag in the blue in more than one outburst during the past 30 years. At the distance of $M 33$, its $M_{V}$ and $\mathrm{M}_{\text {bol }}$ at maximum would be $\sim-8.0$ to $-8.5 \mathrm{mag}$ and therefore it belongs to the class of 'less luminous' LBVs or SDor variables. Recent work by Drissen et al. $(1997,2001)$ has shown that V1 in NGC 2363 should be added to the class of giant eruption LBVs or $\eta$ Carinae-like variables (Humphreys, Davidson \& Smith 1999). V1 has increased more than 4-5 magnitudes in visual light and has stayed at maximun light for about 5 years.

Numerous new LBV or S Dor candidates have appeared in the literature. Several of them are listed here:

HD 168625 - blue hypergiant, post RSG? (Robberto \& Herbst 1998; Sterken et al. 1999; O'Hara et al. 2001; Pasquali et al. 2002)

HD 168607 - blue hypergiant, post RSG? (Sterken et al. 1999)

IRAS 18576+0341 - (Ueta et al. 2001; Pasquali \& Comeron 2002)

FMM 362 in Quintuplet Cluster - (Geballe et al. 2000)

LBV candidate in Cluster 1806-20 - (Eikenberry et al. 2001)

R 85 in LMC - (Massey et al. 2000)

HD 5980 in SMC - (Koenigsberger et al. 2000), but is the physics the same?

B $324=$ UIT 247 in M 33 - (Monteverde et al. 1996; Massey et al. 1996)

B5 = UIT 003 in M 33 - (Kurtev, Corral \& Georgiev 1999)

B 416 in M 33 - (Shemmer et al. 2000)

three UV-bright stars in M 33 - (Massey et al. 1996)

variable in NGC 4414 - (Frattare \& Zurek 1999)

\subsection{The evolutionary path to the $\mathrm{S}$ Dor stage - a controversy solved?}

The lack of observed RSGs above the empirical luminosity boundary, known as the Humphreys-Davidson limit, is considered to be evidence that stars above some initial mass, possibly $50-60 \mathrm{M}_{\odot}$ do not become red supergiants. Therefore the most luminous S Dor-type variables, the 'classical' LBVs, including AG Car, R 127, S Dor itself and several of the Hubble-Sandage variables in M 31 and M 33, have not been red supergiants.

However, ISO observations and the detection of silicates and crystalline minerals in their dusty ejecta, similar to the composition of RSG circumstellar dust, was considered evidence that they had been red supergiants afterall (Waters et al. 1998, 1999; Smith et al. 1998; Voors et al. 2000). Similarities in dust composition do not necessarily mean that a star has been a red supergiant, but only that the composition of the ejecta is similar. S Dor/LBVs are at or just past the end of core H-burning and RSGs are similarly past H-burning. Both represent high mass loss stages with convective/turbulent outer layers, so there may be similarities in the composition of their ejecta. 
In a careful and thorough analytical discussion of the composition of the ejecta from four well-studied stars, AG Car, P Cyg, R 127 and S119, Lamers et al. (2001) conclude that the nebulae were ejected as blue supergiants. The star has not been through a red supergiant stage; however, they suggest a 'temporary red' supergiant phase. The high mass loss episode that forms the nebula or the ejecta will also create a 'false-photosphere', the opaque wind, that looks like a much cooler star. But how cool? The opaque wind limit $\sim 7500 \mathrm{~K}$ in the $\mathrm{S}$ Dor-type transits is not as cool as a true 'red' (M-type) supergiant.

If the massive star actually evolves to the region of the yellow hypergiants (spectral type F-G) and due to the instability at the modified Eddington $/ \Omega$ limit, which it must encounter at its high mass and luminosity, it has a high mass loss episode, then the false photosphere could be as cool as a red supergiant. There are two examples among the cool hypergiants, but they are below the upper luminosity limit and not as luminous as AG Car, etc. The yellow hypergiant $\rho$ Cas has had three recorded 'shell' ejections during which it temporarily had $\mathrm{TiO}$ bands and resembled an M-type star, the most recent in 2000-01 (1946, Bidelman \& McKellar 1957; Beardsley 1961; 1985-86, Boyarchuk et al. 1988; 2000, Lobel et al. 2002). Variable A in M 33, originally an F-type hypergiant, has had the spectrum of an M-type supergiant for 50 years (Humphreys, Jones \& Gehrz 1987)!

But which direction are these cool hypergiants evolving? Are they postmain-sequence stars evolving to the red or are they post-RSGs? A very luminous star evolving bluewards from the RSG can likewise form an opaque wind in a high mass loss episode. In either case, the star is an impostor, and like the SDor/LBVs in eruption, it is not what it appears to be, even temporarily.

\section{The cool hypergiants}

These very luminous evolved stars define the upper luminosity boundary for the cool stars. They are all highly unstable as revealed by their high mass loss rates, infrared excess, photometric and spectroscopic variations and powerful masers in some stars. In a few cases, visible ejecta provide a picture of their mass loss histories and evidence for several high mass loss episodes.

The extreme red supergiant VY CMa is a powerful OH/IR source with a very visible asymmetric nebula (Smith et al. 2001a). Our HST-WFPC2 images reveal several filaments and prominent arcs which may result from multiple asymmetric ejection episodes due to localized events on the surface. Such events probably involve magnetic fields and convection by analogy with solar activity. Other examples of the role of magnetic fields in luminous late-type stars are $\alpha$ Ori with its visible star spots and surface activity, and the OH/IR supergiant VX Sgr with Zeeman splitting of its OH emission (Chapman \& Cohen 1986) and suggestions of a dipole magnetic field (Triglio, Umana \& Cohen 1998).

IRC+10420 may be one of the most important stars in the H-R diagram for understanding the final stages of massive star evolution. With its extraordinary mass loss rate of $3-6 \times 10^{-4} \mathrm{M}_{\odot} \mathrm{yr}^{-1}$, and as one of the warmest known $\mathrm{OH}$ masers and one of the brightest $10-20 \mu \mathrm{m}$ IR sources in the sky, together with an apparent spectral type that has changed from late $\mathrm{F}$ to possibly as early as A2 in 30 years (Oudmaijer et al. 1996; Klochkova et al. 2002) and its complex visible ejecta (Humphreys et al. 1997), it is our best candidate for post-red supergiant 


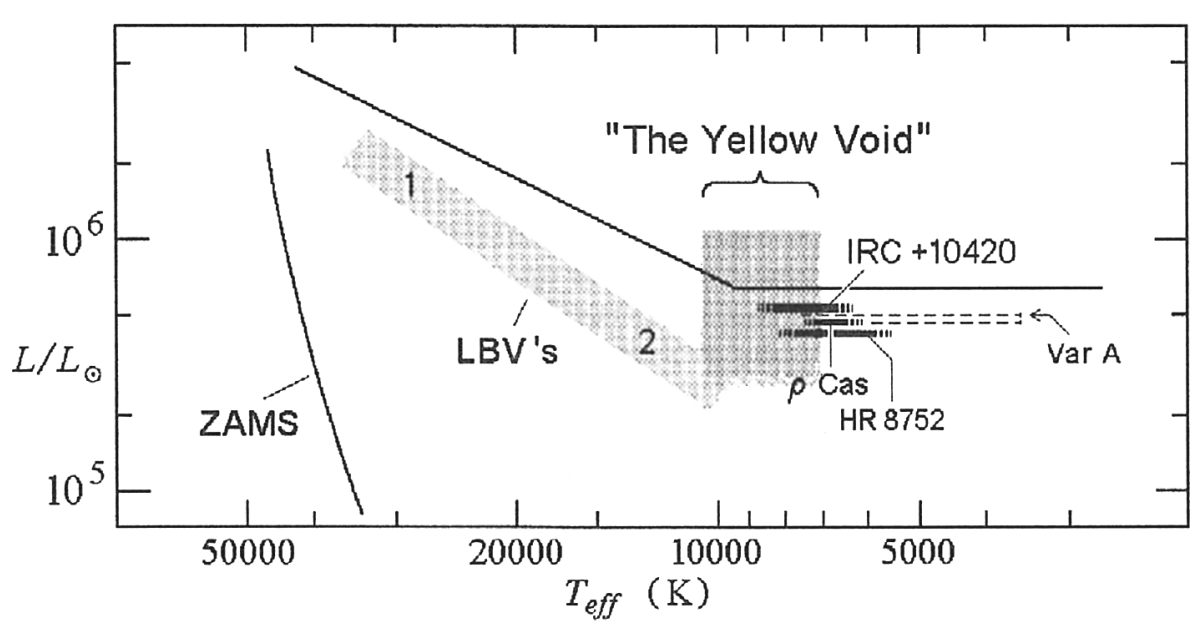

Figure 2. A schematic H-R diagram showing the position of the yellow void with IRC+10 420, Var A in M 33, $\rho$ Cas, and HR 8752 and their range in apparent temperature corresponding to their spectral variations. The shell episodes in $\operatorname{Var} \mathrm{A}$ and $\rho$ Cas are shown as dashed lines. Locations of the classical (1) and less luminous (2) LBVs/S Dor variables in their quiescent state are shown as a band, also known as the the S Doradus instability strip. The solid line is the empirical upper luminosity boundary. From Humphreys, Davidson \& Smith (2002).

evolution. Our HST-sTIS spectroscopy (Humphreys, Davidson \& Smith 2002) of the star's reflected specrum in the ejecta lets us view the star from different directions. Measurements of the double-peaked $\mathrm{H} \alpha$ emission profile show a uniform outflow of gas in a nearly spherical distribution, but its images reveal arcs, rings, jets and knots; evidence for several individual ejections in random directions. Nedoluha \& Bowers (1992) have reported surprisingly strong magnetic fields in the $\mathrm{OH}$ maser regions. Therefore we have suggested that surface activity and magnetic fields may be important in IRC+10420's apparently episodic mass loss events, analogous to VYCMa.

Cornelis de Jager and his collaborators (de Jager \& Nieuwenhuijzen 1997; de Jager 1998; de Jager et al. 2001) have suggested that most if not all of the intermediate temperature (yellow) hypergiants are post-red supergiants. In their blueward evolution they enter a temperature range $(6000-8000 \mathrm{~K})$ of increasing dynamical instability that he calls the 'yellow void', where high mass loss episodes occur (see Figure 2). They specifically address the observed spectroscopic/apparent temperature variations in $\rho$ Cas and HR 8752, which they suggest are caused by the star 'bouncing' against the cool edge of the yellow void in its post-red supergiant evolution. But if the yellow hypergiants. are on blueward evolutionary tracks then their interiors should continue to evolve, independent of their external/surface appearance.

With the very high mass loss rate quoted for IRC+10420 and its temperature range $(7000-8500 \mathrm{~K})$, its wind is optically thick to the continuum. Consequently the observed variations in apparent spectral type and inferred 
temperature are due to changes in the wind and do not necessarily mean that the underlying stellar radius and interior structure are evolving on such a short timescale. We observe its dense wind, but the interior continues to evolve until the 'surface' becomes sufficiently hot to shed its false-photosphere and appear on the blue side of the void. With the recent apparent warming of its dense wind, the recent appearance of strong hydrogen emission, and a possible decline in its mass loss rate, we (Humphreys, Davidson \& Smith 2002) suggest that IRC+10 420 may be in transit across the semi-forbidden region of the yellow void.

Are the spectroscopic variations observed in $\rho$ Cas and HR 8752 due to evolution, to changes in their winds or are they atmospheric? These two hypergiants were observed to be increasing their apparent effective temperatures over the past several years and approaching the temperature regime of the yellow void at $\sim 7000-8000 \mathrm{~K}$ (Israelian, Lobel \& Schmidt 1999), see Figure 2. The normal mass loss rates for these two stars are more than ten times less than for IRC +10420 , so their winds are probably not optically thick. The very thorough discussion of the spectroscopic and photometric variations before, during and after the recent shell episode in $\rho$ Cas by Lobel et al. (2002) demonstrates that the variations and the outburst were caused by a dynamical instability in the atmosphere due to the ionization-recombination of hydrogen. At the time of the outburst, however, the wind was optically thick. After the shell event, the star's apparent surface temperature and spectral type returned to its 'normal' or quiescent state near $\sim 5800 \mathrm{~K}$. This suggests that the previous spectroscopic changes were not due to interior evolution.

Are $\rho$ Cas and HR 8752 post-red supergiants? Unlike IRC+10420, our HSTWFPC2 images of $\rho$ Cas and HR 8752 show no evidence for circumstellar material (Schuster et al. these Proceedings). Thus HR 8752 and $\rho$ Cas may have only recently encountered this unstable region in their blueward evolution. Furthermore, as mentioned above, their normal mass loss rates are at least ten times less than those reported for IRC +10420 . Given their spectroscopic variations and the multiple shell events observed for $\rho$ Cas, I suspect that these stars are on blueward evolutionary tracks.

\subsection{Impostors in the $\mathbf{H}-\mathrm{R}$ diagram?}

At the time of their eruption or outburst, the SDor/LBVs have the spectra of A- or F-type supergiants. We are of course observing their optically thick winds. The underlying star, as we know from their spectra in quiescence, is typically a $20000-30000 \mathrm{~K}$ supergiant.

The A- to F-type spectrum observed for IRC+10 420 is likewise the spectrum of its dense wind. The underlying star could be much warmer.

Three shell events have been observed for the yellow F-type hypergiant $\rho$ Cas during which its opaque wind shows TiO bands and the star looks like an M-type star for several months. Variable A in M 33 is apparently an extreme example of a $\rho$ Cas-like episode. One of the original Hubble-Sandage variables, Var A was the visually brightest star in M 33 until 1951, with the colors and spectrum of an F-type star. It then rapidly declined about three magnitudes. It now has the spectrum of an M-type supergiant and a large infrared excess (Humphreys, Jones \& Gehrz 1987). Unlike $\rho$ Cas, it has not recovered, and has looked like an M-type supergiant for 50 years! 
In all of these examples, the star is not what it appears to be, due to shell events, high mass loss episodes and the production of optically thick winds. Note that, these events all occur in the same temperature regime, near 7000-8000 K, the opaque wind limit, for stars that are already near the modified Eddington limit/ $\Omega$ limit for their masses and luminosities.

\section{The supernovae impostors}

A small subset of the S Dor/LBVs that we have called 'giant eruption' LBVs or ' $\eta$ Carinae'-variables (Humphreys \& Davidson 1994, Humphreys, Davidson \& Smith 1999) experience eruptions during which their total luminosities increase significantly and the star survives. Given the very high luminosities reached during these giant eruptions and because several members of this group have been called supernovae at various times, a catchier name for them is 'supernovae impostors'.

$\eta$ Car is the best known example. Other members of this small group include P Cygni in the 17th century (de Groot 1988; Lamers \& de Groot 1992), SN 1961v in NGC 1058 (Goodrich et al. 1989; Filippenko et al. 1995; van Dyk et al. 2002)), V12 in NGC 2403 (SN 1954j, Tammann \& Sandage 1968), and V1 in NGC 2363 which has been caught in the act by Drissen et al. $(1997,2001)$. The 'Pistol Star' near the Galactic Center (Figer et al. 1998) may be a similar object.

Several candidates have been discovered in the recent supernovae surveys among the TypeIIn's including SN 1997bs (Van Dyk et al. 2000), SN 1999bw (Filippenko, Li \& Modjaz 1999) and SN 2000ch (Filippenko 2000; Wagner et al. 2001).

Confirmation that these are all examples of $\eta$ Carinae-like giant eruptions, is the detection of the remnant star. Of course, we know that $\eta$ Car and P Cygni survived their eruptions and we have recently recovered the image of V12 (Smith, Humphreys \& Gehrz 2001). The nature of the SN 1961v eruption and the detection of the surviving star is more controversial (Filippenko et al. 1995; Stockdale et al. 2001; Van Dyk et al. 2002).

The increasing number of objects displaying characteristics similar to $\eta$ Car's great eruption suggests that these 'giant eruptions' are not so very rare, therefore raising some interesting questions about how the very most massive stars end their lives.

\section{References}

Beardsley, W.A. 1961, ApJS 5, 381

Bidelman, W.P., McKellar, A. 1957, PASP 69, 31

Boyarchuk, A.A., Boyarchuk, M.E., Petrov, P.P. 1988, in: Hanni \& Tuominen (eds.), Proc. of the Sixth Soviet-Finnish Astronomical Meeting, Tartu Astrofuus Obs. Teated 92, 40

Chapman, J.M., Cohen, R.J. 1986, MNRAS 220, 513

Conti, P.S. 1984, in: A. Maeder \& A. Renzini (eds.), Observational Tests of Stellar

Evolution Theory, Proc. IAU Symp. No. 105 (Dordrecht: Reidel), p. 233

Davidson, K. 1987, ApJ 317, 760

de Groot, M. 1988, Irish Astron. J. 18, 163

de Jager, C. 1998, The A\&A Review 8, 145 
de Jager, C., Lobel, A., Nieuwenhuijzen, H., Stothers, R. 2001, MNRAS 327, 452

de Jager, C., Nieuwenhuijzen, H. 1997, MNRAS 290, L50

Drissen, L., Roy, J.-R., Robert, C. 1997, ApJ 474, L35

Drissen, L., Crowther, P.A., Smith, L.J., et al. 2001, ApJ 546, 484

Eikenberry, S.S., Matthews, K., Garske, M.A., et al. 2001, BAAS 199, 9605

Figer, D.F., Najarro, F., Morris, M., McLean, I.S., Geballe, T.R. 1998, ApJ 506, 384

Filippenko, A.V. 2000, IAU Circular No. 7421

Filippenko, A.V., Barth, A.J., Bower, G.C., Ho, L.C., et al. 1995, AJ 110, 2261

Filippenko, A.V., Li, W.D.,, Modjaz, M. 1999, IAU Circular No. 7152

Frattare, L.M., Zurek, D.R. 1999, BAAS 194, 8202

Geballe, T.R., Najarro, F., Figer, D.F. 2000, ApJ 530, 97

Goodrich, R.W., Stringfellow, G.S., Penrod, G.D., Filippenko, A.V. 1989, ApJ 342, 908

Humphreys, R.M. 1999, in: B. Wolf, O. Stahl \& A.W. Fullerton (eds.), Variable and Non-spherical Stellar Winds in Luminous Hot Stars, Proc. IAU Coll. No. 169 (Berlin: Springer), p. 243

Humphreys, R.M., Davidson, K. 1979, ApJ 232, 409

Humphreys, R.M., Davidson, K. 1984, Science 223, 243

Humphreys, R.M., Davidson, K. 1994, PASP 106, 1025

Humphreys, R.M., Davidson, K., Smith, N. 1999, PASP 111, 1124

Humphreys, R.M., Davidson, K., Smith, N. 2002, AJ 124, 1026

Humphreys, R.M., Jones, T.J., Gehrz, R.D., 1987, AJ 94, 3151

Humphreys, R.M., Smith, N., Davidson, K., Jones, T.J., et al. 1997, AJ 114, 2778

Israelian, G., Lobel, A., Schmidt, M. 1999, ApJ (Letters) 523, L145

Jones, T.J., Humphreys, R.M., Gehrz, R.D., et al. 1993, ApJ 411, 323

Klochkova, V.G., Yushkin, M.V., Chentsov, E.L., et al. 2002, Astron. Reports 46, 139

Koenigsberger, G., Georgiev, L., Barbá, R., et al. 2000, ApJ 542, 428

Kurtev, R., Sholukhova, O., Borissova, J., Georgiev, L. 2001, RMxAA 37, 57

Kurtev, R., Corral, L.J., Georgiev, L. 1999, A\&A 349, 796

Lamers, H., de Groot, M. 1992, A\&A 257, 153

Lamers, H., Nota, A., Panagia, N., Smith, L.J., Langer, N. 2001, ApJ 551, 764

Lobel, A., Dupree, A.K., Stefanik, R.P., Torres, G., et al. 2002, ApJ in press

Massey, P., Bianchi, L., Hutchings, J.B., Stecher, T.P. 1996, ApJ 471, 203

Massey, P., Waterhouse, E., DeGoia-Eastwood, K. 2000, AJ 119, 2214

Monteverde, M.I., Herrero, A., Lennon, D.J., Kudritzki, R.-P. 1996, A\&A 312, 24

Nedoluha, G.E., Bowers, P.F. 1992, ApJ 392, 249

O'Hara, T.B., Meixner, M., Speck, A.K., et al. 2001, BAAS 199, 13521

Oudmaijer, R.D., Groenewegen, M.A.T., et al. 1996, MNRAS 280, 1062

Pasquali, A, Comerón, F. 2002, A\&A 382, 1005

Pasquali, A, Nota, A., Smith, L.J., et al. 2002, AJ 124, 1625

Robberto, M., Herbst, T.M. 1998, ApJ 498, 400

Shemmer, O., Leibowitz, E., Szkody, P. 2000, MNRAS 311, 698

Smith, L.J., Nota, A., Pasquali, A., et al. 1998, ApJ 503, 278

Smith, N., Humphreys, R.M., Davidson, K., et al. 2001, AJ 121, 1111

Smith, N., Humphreys, R.M., Gehrz, R.D. 2001, PASP 113, 692

Sterken, C., Arentoft, T., Duerbeck, H.W., Brogt, E. 1999, A\&A 349, 532

Stockdale, C.J., Rupen, M.P., Cowan, J.J., Chu, Y.-H., Joens, S.S. 2001, AJ 122, 283 
Tammann, G.A., Sandage, A. 1968, ApJ 151, 825

Triglio, C., Umana, G., Cohen, R.J. 1998, MNRAS 297, 497

Ueta, T., Meixner, M., Dayal, A., et al. 2001, ApJ 548, 1020

Van Dyk, S.D., Peng, C.Y., King, J.Y., et al. 2000, PASP 112, 1532

Van Dyk, S.D., Filippenko, A.V., Li, W.D. 2002, PASP 114, 700

van Genderen, A.M. 2001, A\&A 366, 508

Voors, R.H.M., Waters, L.B.F.M., de Koter, A., et al. 2000, A\&A 356, 501

Wagner, R.M., Vrba, F.J, Henden, A.A., et al. 2001, BAAS 32, 1474

Waters, L.B.F.M., Morris, P.W., Voors, R.H.M., Lamers, H., Trams, N.R. 1998, in: L.B.F.M. Waters, C. Waelkens, K.A. van der Hucht \& P.A. Zaal (eds.), ISO's View on Stellar Evolution, Ap\&SS 255, 179

Waters, L.B.F.M., Voors, R.H.M., Morris, P.W., Trams, N.R., de Koter, A., Lamers, H. 1999, in: B. Wolf, O. Stahl \& A.W. Fullerton (eds.), Variable and Nonspherical Stellar Winds in Luminous Hot Stars, Proc. IAU Coll. No. 169 (Berlin: Springer), p. 381

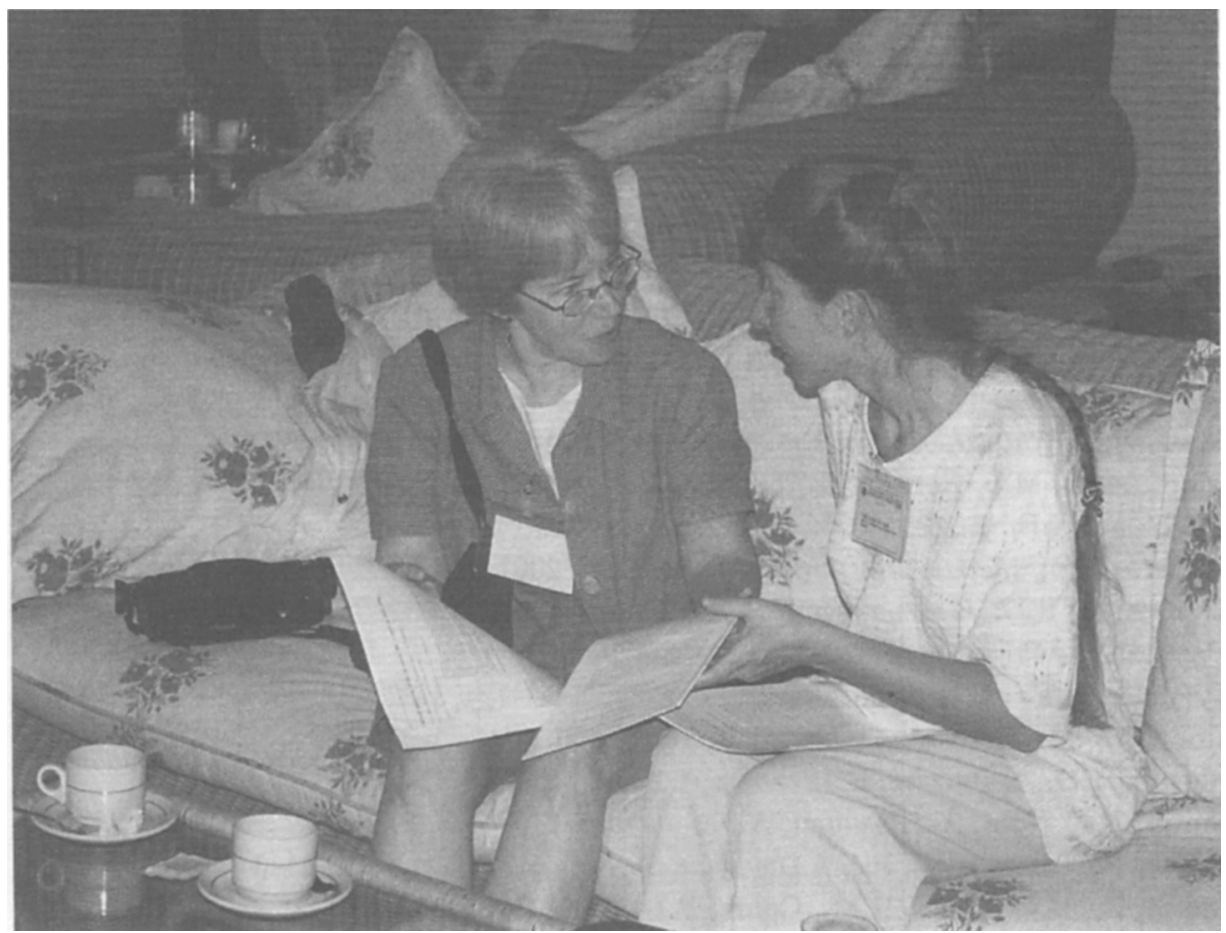

Roberta Humphreys and Olga Sholukhova, comparing notes 